
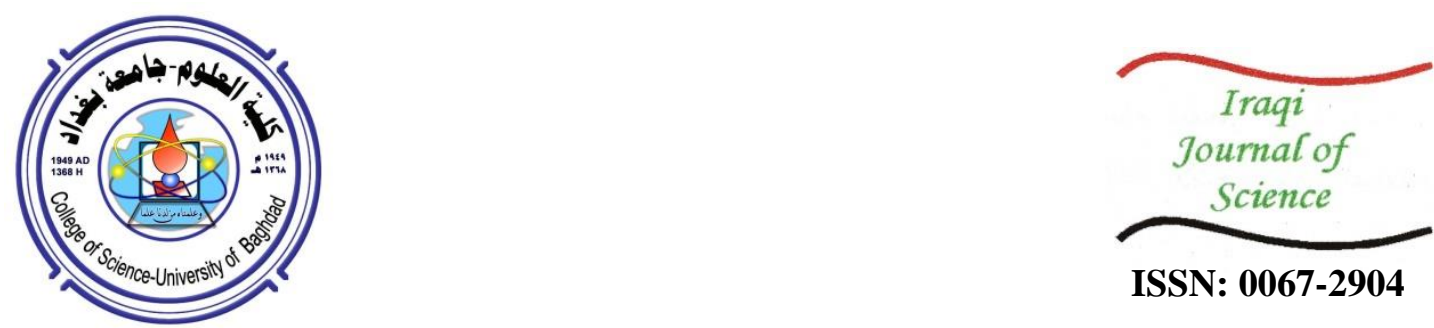

\title{
On Soft bc-Open Sets in Soft Topological Spaces
}

\author{
Saif Z. Hameed*, Adiya K. Hussein \\ Department of Mathematics, College of Basic Education Mustansiriyah University, Baghdad, Iraq
}

Received: 5/1/ 2020

Accepted: $15 / 3 / 2020$

\begin{abstract}
In this paper, we offer and study a novel type of generalized soft-open sets in soft topological spaces, named soft bc-open sets. Relationships of this set with other types of generalized soft-open sets are discussed, definitions of soft $b c-$ neighborhood, soft bc- closure and soft bc- interior are introduced, and its properties are investigated. Also, we introduce and explore several characterizations and properties of this type of sets.
\end{abstract}

Keywords: soft b-open set, soft bc-open set, soft bc- nbd, soft bc-interior, soft bcclosure.

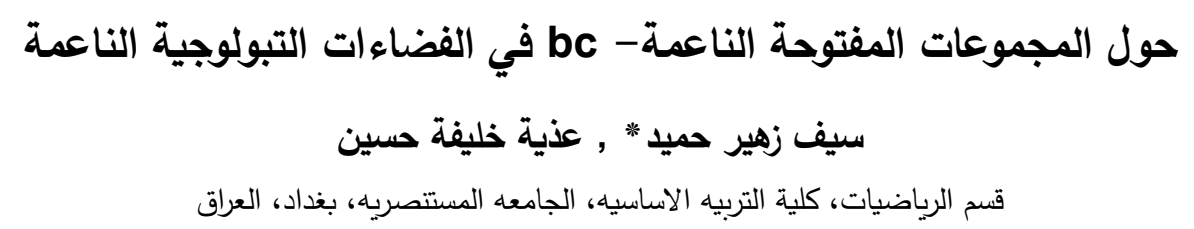

$$
\begin{aligned}
& \text { الخلاصة } \\
& \text { في هذا البحث قدمنا ودرسنا نوع جديد من المجموعات المفتوحة الناعمة اسميناها (المجموعات المفتوحة }
\end{aligned}
$$

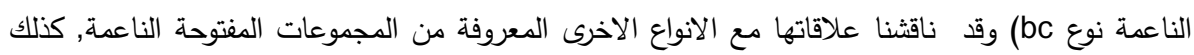

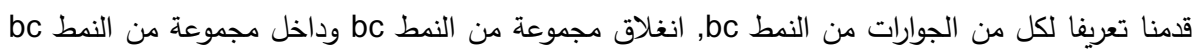

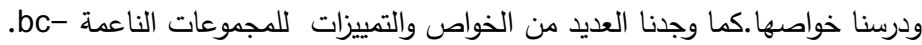

\section{Introduction and Preliminaries}

The concept of soft set theory was instigated and applied by Molodtsov [1,2] as a mathematical device for dealing with uncertainties. In a previous work [3], Shaber and Naz defined soft topological spaces and soft open sets. Soft $\beta$ - open sets were introduced and studied by several authors, including the soft $\alpha$ - open [4], soft preopen [5], soft semi open [4], and soft regular open sets [6]. . In another study [7], Akdag and Ozkan realized the soft b- open sets and soft continuity. The concept of bc- open sets was introduced by Ibrahim [8].

Let $(Z, \tau, \mathrm{A})$ be a soft topological space, where $\mathrm{A}$ is any set of parameters. The soft closure (resp. soft interior) [9] of a soft set $(P, A)$ is denoted by $(\operatorname{cl}(P, A)($ resp. $\operatorname{int}(P, A))$. A subset $(P, A)$ is said to be a $\beta$-open [4]( resp. soft $\alpha$-open[5], soft preopen[4], soft semi-open[6] and soft regular) set, if: $(P, A) \subset \operatorname{cl}(\operatorname{int}(\operatorname{cl}((P, A)))(\operatorname{resp} .(P, A) \subset \operatorname{int}(\operatorname{cl}(\operatorname{int}((P, A)))),(P, A) \subset$ $\operatorname{int}(\operatorname{cl}((P, A))),(P, A) \subset \operatorname{cl}(\operatorname{int}((P, A)))$ and $(P, A)=\operatorname{int}(\operatorname{cl}((P, A)))$.

We denote the family of all soft sets over $\mathrm{X}$ by $\operatorname{SS}(Z, A)$.

Definition 1.1[3]. Let $\tau$ be a collection of soft open sets over $Z$, then $\tau$ is said to be soft topological space if (1) $\widetilde{\varnothing}$ and $\tilde{X}$ belong to $\tau$, (2) The union of any subcollection

*Email: saif.zuhar.edbs@uomustansiriyah.edu.iq 
of soft sets of $\tau$ belongs to $\tau$, and (3) the intersection of any two soft sets in $\tau$ belongs to $\tau$. We named the triple $(Z, \tau, \mathrm{A})$ by $\boldsymbol{S T S}$.

Definition 1.2 [9]. The soft set $(P, A) \in S S(Z, A)$ is called a soft point in $Z$, denoted by $e_{L}$, if for the element $e \in A, F(e) \neq \varnothing$ and $F\left(e^{\prime}\right)=\emptyset$ for all $e^{\prime} \in A \backslash\{e\}$. The set of all soft points of $Z$ is denoted by $S P(Z)$.

Definition 1.3 [7]. If $(P, A) \in S S(Z, A)$ then it is called

(1) Soft $\mathrm{b}-$ open set (briefly $s \mathrm{~b}-$ open set), iff $(P, A) \subset \operatorname{int}(\operatorname{cl}((P, A))) \cup \operatorname{cl}(\operatorname{int}((P, A)))$.

(2) Sof tb - closed (briefly, $s \mathrm{~b}-\operatorname{closed})$ set, iff $(P, A) \supset \operatorname{int}(\operatorname{cl}((P, A))) \cap \operatorname{cl}(\operatorname{int}((P, A)))$.

Definition $1.4[6,7]$. Let $(P, A)$ be a soft set of a STS $(Z, \tau, \mathrm{A})$, then

(1) Soft semi - interior of a $\operatorname{soft} \operatorname{set}(P, A)$ in $Z$ is denoted by $\operatorname{sint}((P, A))=\cup\{(W, A):(W, A)$ is a soft semi-open set and $(W, A) \subset(P, A)\}$.

(2) Soft semi - closure of a soft set $(P, A)$ in $Z$ is denoted by $\operatorname{sScl}((P, A))=\cap\{(L, A):(L, A)$ is a soft semi-closed set and $(P, A) \subset(L, A)\}$.

(3) Soft $b$ - interior of a $\operatorname{soft} \operatorname{set}(P, A)$ in $Z$ is denoted by $\operatorname{sbint}((P, A))=\cup\{(W, A):(W, A)$ is a soft $\mathrm{b}-$ open set and $(W, A) \subset(P, A)\}$.

(4) Soft $b-$ closure of a soft set $(P, A)$ in $Z$ is denoted by $\operatorname{sbcl}((P, A))=\cap\{(L, A):(L, A)$ is a soft $\mathbf{b}-$ closed set and $(P, A) \subset(L, A)\}$.

Clearly, $\operatorname{sbcl}(P, A)$ (resp. $\operatorname{sScl}(P, A)$ is the smallest soft b-closed (resp. soft semi-closed) set over $Z$ which contains $(P, A)$, and sbint $((P, A))$ (resp. sSint $((P, A)))$ is the largest soft $b$-open (resp. semiopen) set over $Z$ which is contained in $(P, A)$.

We will denote the family of all soft $b$-open (resp., soft semi-open) sets and soft b-closed ((resp., soft semi-closed) sets of a soft topological space by $b O(Z)$ (resp., $\operatorname{SSO}(Z) \operatorname{SbC}(Z)$ (resp., $\operatorname{SSC}(Z)$ ).

Definition $1.5[10]$. Let $(Z, \tau, \mathrm{A})$ be a STS. and $x, y \in Z$, such that $x \neq y$. If there exist soft open sets $(P, A)$ and $(S, A)$, such that $x \in(P, A), y \notin(S, A), y \in(S, A)$, and $x \notin(P, A)$, then $(Z, \tau, \mathrm{A})$ is called a soft $T_{1}$-space.

Theorem 1.6 [10]. Let $(Z, \tau, \mathrm{E})$ be $\boldsymbol{S T S}$. Then each soft point is a soft closed if and only if $(Z, \tau, \mathrm{A})$ is a soft $T_{1}$-space.

Definition 1.7 [11]. An $\boldsymbol{S T S}(Z, \tau, \mathrm{A})$ is called a soft locally indiscrete, if every soft open set over $Z$ is a soft closed set over $Z$.

\section{Soft bc - open sets}

Now, we give a new family of soft $b$ - open sets named soft $b c$ - open sets in an STS and study some of its basic properties.

Definition 2.1 A subset $(P, A)$ of $\operatorname{STS}(Z, \tau, \mathrm{E})$ is named soft $b c-$ open (sbc-open) if, for any $x \in(P, A) \in S b O(Z)$, there is a soft closed set $(\mathrm{S}, A)$, such that $x \in(\mathrm{S}, A) \subset(P, A)$. The complement of $(P, A)$ is named soft $b c-$ closed $(s b c-$ closed $)$.

The collection of all soft $b c$ - open sets in $Z$ is denoted by $\operatorname{SbcO}(Z)$ and the collection of all soft $b c-$ closed sets in $Z$ is named $\operatorname{Sbc} C(Z)$.

Theorem 2.2 A soft subset $(P, A)$ of $\operatorname{STS}(Z, \tau, \mathrm{A})$ is soft $b c$ - open iff $(P, A)$ is $s b$-open and it is a union of soft closed sets.

Proof. $(\Rightarrow)$ Let $(P, \mathrm{~A})$ be a soft $b c-$ open set. Then $(P, A)$ is $s b$ - open set and for each $x \in(P, A)$ there is a soft closed set $(L, A)$, such that $x \in(L, A) \subseteq(P, A)$. Then we get $\bigcup\{x\}_{x \in(P, A)}=(P, A) \subseteq$ $(L, A) \subseteq(P, A)$. Thus, $(P, A)=\bigcup(L, A)$, where $(P, A)$ is a soft closed set for each $x \in(P, A)$.

$(\Leftarrow)$ Direct form the definition of soft $b c-$ open.

Corollary 2.3 For a STS $(Z, \tau, A)$, if $(L, A)$ is $s b$-open set over $\mathrm{X}$, then $(L, A)$ is an $s b c-$ open if $(L, A)$ is a soft closed set.

Proposition 2.4 A soft subset $(\mathrm{H}, A)$ of an $\boldsymbol{S T S}(Z, \tau, \mathrm{A})$ is $s b c$ - closed if and only if $(\mathrm{H}, A)$ is a soft $b$-closed set and it is an intersection of soft open sets.

Proof. It is obvious.

Remark 2.5 Every $s b c$-open set of a space $Z$ is soft $b$-open, but the converse is not true in general, as shown by the following example.

Example 2.6 Let $Z=\left\{v_{1}, v_{2}, v_{3}\right\}, A=\left\{e_{1}, e_{2}, e_{3}\right\}$ and

$\tau=\left\{\widetilde{\varnothing}, \widetilde{Z},\left(P_{1}, A\right),\left(P_{2}, A\right),\left(P_{3}, A\right),\left(P_{4}, A\right)\right\}$

where $\left(P_{1}, A\right),\left(P_{2}, A\right),\left(P_{3}, A\right),\left(P_{4}, A\right)$ are soft sets over $Z$, defined as follows: 


$$
\begin{aligned}
& \left(P_{1}, A\right)=\left\{\left(e_{1}, \widetilde{Z}\right),\left(e_{2},\left\{v_{2}, v_{3}\right\}\right),\left(e_{3},\left\{v_{1}, v_{2}\right\}\right)\right\}, \\
& \left(P_{2}, A\right)=\left\{\left(e_{1}, \widetilde{\varnothing}\right),\left(e_{2},\left\{v_{1}\right\}\right),\left(e_{3},\left\{v_{3}\right\}\right)\right\}, \\
& \left(P_{3}, A\right)=\left\{\left(e_{1},\left\{v_{2}\right\}\right),\left(e_{2},\left\{v_{1}, v_{3}\right\}\right),\left(e_{3},\left\{v_{3}\right\}\right)\right\}, \\
& \left(P_{4}, A\right)=\left\{\left(e_{1},\left\{v_{2}\right\}\right),\left(e_{2},\left\{v_{3}\right\}\right),\left(e_{3}, \widetilde{\varnothing}\right)\right\} .
\end{aligned}
$$

Then, $\tau$ defines a soft topology on $Z$.

The soft closed sets are $\widetilde{Z}, \widetilde{\emptyset},\left(P_{1}, A\right)^{c},\left(P_{2}, A\right)^{c},\left(P_{3}, A\right)^{c},\left(P_{4}, A\right)^{c}$,

where $\left(P_{1}, A\right)^{c}=\left\{\left(e_{1}, \widetilde{\emptyset}\right),\left(e_{2},\left\{v_{1}\right\}\right),\left(e_{3},\left\{v_{3}\right\}\right)\right\}=\left(P_{2}, A\right)$

$\left(P_{2}, A\right)^{c}=\left\{\left(e_{1}, \widetilde{Z}\right),\left(e_{2},\left\{v_{2}, v_{3}\right\}\right),\left(e_{3},\left\{v_{1}, v_{2}\right\}\right)\right\}=\left(P_{1}, A\right)$

$\left(P_{3}, A\right)^{c}=\left\{\left(e_{1},\left\{v_{1}, v_{3}\right\}\right),\left(e_{2},\left\{v_{2}\right\}\right),\left(e_{3},\left\{v_{1}, v_{2}\right\}\right)\right\}$,

$\left(P_{4}, A\right)^{c}\left\{\left(e_{1},\left\{v_{1}, v_{3}\right\}\right),\left(e_{2},\left\{v_{2}, v_{3}\right\}\right),\left(e_{3}, \widetilde{Z}\right)\right\}$.

The family of $\operatorname{SbOS}(Z)=\left\{\left(P_{1}, A\right),\left(P_{2}, A\right),\left(P_{3}, A\right),\left(P_{4}, A\right)\right\}$.

The family of $\operatorname{SbcOS}(Z)=\left\{\left(P_{1}, A\right),\left(P_{2}, A\right),\left(P_{3}, A\right)\right\}$.

Then $\left(P_{4}, A\right) \in \operatorname{SbOS}(Z)$, but $\left(P_{4}, A\right) \notin \operatorname{SbcOS}(Z)$.

By Remark (2) [8] and the above Remark (2.5), we have the following implications:

Soft regular set $\Rightarrow$ Soft -open set $\Rightarrow$ Soft $\alpha$-open set $\Rightarrow$ Soft semi -open set

$\Downarrow$

$$
\text { Soft pre -open set } \Rightarrow \begin{gathered}
\text { Soft } \bar{b}-\text { open set } \Rightarrow \text { Soft } \beta \text {-open set } \\
\Uparrow
\end{gathered}
$$

Soft $b c$-open set

Proposition 2.7An arbitrary union of $s b c-$ open sets is $s b c-o p e n$ set.

Proof. Suppose that $\left\{(P, A)_{\lambda}: \lambda \in \Delta\right\}$ is a family of soft $b c-$ open $\operatorname{sets} \operatorname{in}(Z, \tau, A)$. Then $(P, A)_{\lambda}$ is soft $b$-open set for each $\lambda \in \Delta$. So, $\bigcup(P, A)_{\lambda}$ is soft $b$-open. Let $x \in \cup\left\{(P, A)_{\lambda}: \lambda \in \Delta\right\}$, so $x \in(L, A)_{\lambda}$ for some $\lambda \in \Delta$. Since $(P, A)_{\lambda}$ is soft $b-$ open for each $\lambda$, then there is a soft closed set $(L, A)$ such that

$x \in(L, A) \subset(P, A)_{\lambda} \subset \cup\left\{(P, A)_{\lambda}: \lambda \in \Delta\right\}$, so $x \in(L, A) \subset \cup\left\{(P, A)_{\lambda}: \lambda \in \Delta\right\}$. Therefore, $\cup\left\{(P, A)_{\lambda}\right.$ : $\lambda \in \Delta\}$ is soft $b c-$ open set.

Now we show that the intersection of two $s b c$ - open sets is not necessarily sbc-open.

Example 2.8. Let the $\boldsymbol{S T S}(Z, \tau, \mathrm{A})$ as in Example 2.6, then $\left(P_{1}, A\right) \in \operatorname{SbcO}(Z)$ and $\left(P_{3}, A\right) \in$ $\operatorname{SbcO}(Z)$, but $\left(P_{1}, A\right) \cap\left(P_{3}, A\right)=\left(P_{4}, A\right) \notin \operatorname{SbcO}(Z)$.

Remark 2.9. From the above example we notice that the family of all sbc-open subset of a space $Z$ is a supra topology and thus it is not a topology in general.

The following result gives a condition under which the family of all $\mathrm{s} b c$ - open sets became a topology on Z.

Proposition 2.10. If the collection $S b O(Z)$ is a topology on $Z$, then $\operatorname{SbcO}(Z)$ is also a topology on $Z$.

Proof. It is clear that $\widetilde{\emptyset}, \widetilde{Z} \in \operatorname{Sbc} O(Z)$ and, by Proposition 2.7 , the union of any subset of $\operatorname{SbcO}(Z)$ is $s b c-o p e n$. Now, let $(P, A)$ and $(\mathrm{S}, A)$ be two $s b c-$ open sets, then $(P, A)$ and $(P, A)$ are soft $b-$ open sets. Since $S b O(Z)$ is a topology on $Z$, so $(P, A) \cap(\mathrm{S}, A)$ is soft $b$-open. If $x \in(\mathrm{S}, A) \cap(P, A)$, then $x \in(P, A)$ and $x \in(\mathrm{S}, A)$. So there exist two soft closed sets $(L, A)$ and $(K, A)$, such that $x \in(L, A) \subset(\mathrm{S}, A)$ and $x \in(K, A) \subset(P, A)$. This implies that $x \in(L, A) \cap(K, A) \subset(\mathrm{S}, A) \cap(P, A)$. Since any intersection of soft closed sets is soft closed, then $(L, \mathrm{E}) \cap(K, A)$ is a closed set. Thus, $(P, A) \cap(\mathrm{S}, A)$ is $s b c$-open set.

Theorem 2.11 A soft set $(P, A)$ of a STS $(Z, \tau, A)$ is a soft $b c$-open set iff, for each $x \in(P, A)$, there is a soft $s b c-$ open set $(\mathrm{S}, A)$ such that $x \in(\mathrm{S}, A) \subseteq(P, A)$.

Proof. Suppose that $(P, A)$ is soft $b c$-open in the space $Z$, then for each $x \in(P, A)$, put $(P, A)=$ $(\mathrm{S}, A)$ is $s b c-$ open set containing $x$ such that $x \in(P, A) \subseteq(\mathrm{S}, A)$.

Conversely, assume that for any $x \in(P, A)$, there is a $s b c$-open set $(\mathrm{S}, A)$ such that $x \in(\mathrm{S}, A) \subseteq$ $(P, A)$. Thus, $(P, A)=\mathrm{U}(\mathrm{S}, A)_{x}$ where $(\mathrm{S}, A)_{x} \in \operatorname{Sbc} O(Z)$ for each $x$. Hence, $(P, A)$ is $s b c-$ open set. 
Theorem 2.12 Let $(Z, \tau, A)$ be soft $T_{1}$-space, then $(P, A)$ is $s b$-open set iff $(P, A)$ is a soft bc-open.

Proof Suppose that $(Z, \tau, A)$ is soft $T_{1}$ - space and $(P, A)$ is $s b-$ open set. If $(P, A)=\widetilde{\emptyset}$, then $(P, A) \in \operatorname{sbc}(Z)$. If $(P, A) \neq \widetilde{\emptyset}$, let $x \in(P, A)$. Since $(Z, \tau, A)$ is soft $T_{1}-$ space, then by Theorem 1.6, each soft point is a closed set and, hence, $x \in\{x\} \subset(P, A)$. Therefore, $(P, A)$ is an $s b c-$ open, thus $S b O(Z) \subset S b c O(Z)$. But $S b c O(Z) \subset S b O(Z)$. Hence, $S b O(Z)=S b c O(Z)$.

Proposition 2.13 If $(Z, \tau, A)$ is soft locally indiscrete, then $S S O(Z) \subset S b c O(Z)$.

Proof. Let $(P, A)$ be any soft subset of $\operatorname{STS}(Z, \tau, A)$ and $(P, A) \in S S O(Z)$, if $(P, A)=\widetilde{\emptyset}$, then $(P, A) \in \operatorname{SbcO}(Z)$. If $(P, A) \neq \widetilde{\emptyset}$, then $(P, A) \subset \operatorname{cl}(\operatorname{int}((P, A)))$. Since $(Z, \tau, A)$ is soft locally indiscrete, then $\operatorname{int}(P, A)$ is soft closed, so $\operatorname{int}(P, A) \subset(P, A)$. This implies that for each $x \in(P, A), x \in(\operatorname{int}(P, A)) \subset(P, A)$. Therefore, $(P, A)$ is $\operatorname{sbc}-$ open set. Hence $\operatorname{SSO}(Z) \subset$ $\operatorname{SbcO}(Z)$.

Theorem 2.14 Let $\left\{(P, A)_{\alpha}: \alpha \in \Delta\right\}$ be a collection of sbc-closed sets in a soft topological space $(Z, \tau, A)$. Then $\cap\left\{(P, A)_{\alpha}: \alpha \in \Delta\right\}$ is soft $b c-$ closed.

Proof. The proof follows from Proposition 2.7.

\section{Some Properties of Soft $b c-$ Open Sets}

In this section, we provide some soft topological operations on oft sets and discuss its properties.

Definition 3.1 Let $(Z, \tau, A)$ be an STS and $x \in \widetilde{Z}$.Then, a soft set $(P, A)$ is said to be soft $b c-$ neighborhood (briefly, soft $b c-n b h)$ of $x$, if there exists a soft $b c-$ open set $(K, A)$ over $Z$ such that $x \in(K . A) \subset(P, A)$.

Proposition 3.2 For an STS $(Z, \tau, A)$, a soft set $(P, A)$ is $s b c$-open iff it is a soft $b c-$ neighborhood of each of its points.

Proof. Let $(P, A) \subset \widetilde{Z}$ be a soft $s b c-$ open set, since for every $x \in(P, A), x \in(P, A) \subset(P, A)$ and $(P, A)$ is $s b c-o p e n$, this shows that $(P, A)$ is a soft $b c-$ neighborhood of each of its points.

Conversely, suppose that $(P, A)$ is a soft $b c-$ neighborhood of each of its points. Then for each $x \in(P, A)$, there exists $(\mathrm{S}, A)_{x} \in \operatorname{Sbc} O(Z)$ such that $(\mathrm{S}, A)_{x} \subset(P, A)$. Then $(P, A)=\bigcup\left\{(\mathrm{S}, A)_{x}\right.$ : $x \in(P, A)\}$. Since each $(\mathrm{S}, A)_{x}$ is $s b c-o p e n$. It follows that $(P, A)$ is $s b c-o p e n$.

Proposition 3.3 Every soft $b c-$ neighborhood of a point is soft $b-$ neighborhood.

Proof. It is obvious from the fact that every $s b c-$ open set is $s b-o p e n$.

Definition 3.4 Let $(P, A)$ be soft set of a STS $(Z, \tau, A)$, then a point $x \in Z$ is called soft $b c-$ interior point of $(P, A)$, if there exists an $s b c$-open set $(U, A)$ such that $x \in(U, A) \subset(P, A)$. The set of all $s b c$-interior points of $(P, A)$ is denoted by $\operatorname{sbc} \operatorname{lnt}(P, A)$.

Proposition 3.5 Let $(P, A)$ be a soft set of $Z$, then $\operatorname{sbcInt}(P, A) \subset \operatorname{sbint}(P, A)$.

Proof. Since $s b c-$ open set iss $b-$ open, so the proof holds.

Definition 3.6 Let $(P, A)$ be asoft set of a STS $(Z, \tau, A)$, then the soft $b c$ - closure of $(P, A)$, denoted by $\operatorname{sbcCl}(P, A)$, is the intersection of all $s b c-$ closed sets containing $(P, A)$.

In the following theorem we provide some properties of $s b c$-interior of a soft set.

Theorem 3.7 Let $(Z, \tau, A)$ be STS and let $(P, A)$ and $(\mathrm{M}, A)$ be soft sets over $Z$. Then

1) $\operatorname{sbcInt}(P, A)$ is the union of all $s b c-$ open sets which are contained in $(P, A)$.

2) $\operatorname{sbcInt}(P, A)$ is $s b c-$ open set in $Z$.

3) $(P, A)$ is $\operatorname{sbc}-$ open iff $(P, A)=\operatorname{sbc} \operatorname{Int}(P, A)$.

4) $\operatorname{sbcInt}(\operatorname{sbcInt}(P, A))=\operatorname{sbcInt}(P, A)$.

5) $\operatorname{sbcInt}(\widetilde{\varnothing})=\widetilde{\emptyset}$ and $\operatorname{sbcInt}(\widetilde{Z})=\widetilde{Z}$.

6) $\operatorname{sbcInt}(P, A) \subset(P, A)$.

7) If $(P, A) \subset(\mathrm{M}, A)$, then $\operatorname{sbcInt}(P, A) \subset \operatorname{sbcInt}(\mathrm{M}, A)$.

8) If $(P, A) \cap(\mathrm{M}, A)=\widetilde{\emptyset}$, then $\operatorname{sbcInt}(P, A) \subset \operatorname{sbcInt}(\mathrm{M}, A)$.

9) $\operatorname{sbcInt}(P, A) \cup \operatorname{sbcInt}(\mathrm{M}, A) \subset \operatorname{sbcInt}((P, A) \cup(\mathrm{M}, A))$.

10) $\operatorname{sbcInt}((P, A) \cap(\mathrm{M}, A)) \subset \operatorname{sbcInt}(P, A) \cap \operatorname{sbcInt}(\mathrm{M}, A)$.

Proof. The proofs of these facts are easy, so we will only prove the point number 7:

Suppose that $x \in Z, x \in \operatorname{sbcInt}(P, A)$, then by Definition 3.4, there is a set $(U, A)$ such that $x \in(U, A) \subset(P, A) \subset(P, A)$, thus $x \in \operatorname{sbcInt}(P, A)$. 
Theorem 3.8 Let $(P, A)$ be any soft set in a $\operatorname{STS}(Z, \tau, A)$, then $x \in \operatorname{sbcCl}(Z)$ if and only if $(P, A) \cap(U, A)=\emptyset$ for any $s b c-$ open set $(U, A)$ containing $x$.

Proof. $\Rightarrow) \quad$ let $\quad x \in \operatorname{sbcCl}(P, A)$. Suppose that $(P, A) \cap(U, A)_{x}=\emptyset$, where $(U, A)_{x} \in$ $\operatorname{sbc} O(Z)$ containing $x$. Hence, $(P, A) \subset(U, A)_{x}^{c}$, where $(U, A)_{x}^{c} \in \operatorname{sbc} C(P, A)$. Hence, $x \notin \operatorname{sbcCl}(P, A)$, which is a contradiction.

$(\Leftarrow) \quad$ Let $x \notin \operatorname{sbcCl}(P, A)$, then $x \notin \cap(L, A)$, where $(L, A) \in \operatorname{sbcC}(Z)$ and $(P, A) \subset(L, A)$ for each $(L, A)$. Hence, $x \in(\cap(L, A))^{c}$, where $(\cap(L, A))^{c} \in \operatorname{sbcO}(Z)$ containing $\mathrm{x}$. Now, we have $(P, A) \cap(\cap(L, A))^{c} \subset(\cap(L, A)) \cap(\cap(L, A))^{c}=\emptyset$.

Theorem 3.9 Let $(P, A)$ be any soft subset of an $\boldsymbol{S T S}(Z, \tau, A)$ if $(P, A) \cap(L, A) \neq \emptyset$ for any soft closed $\operatorname{set}(L, A)$ containing $x$, then $x \in \operatorname{sbcCl}(P, A)$.

Proof. Suppose that $(\mathrm{U}, A)_{x} \in \operatorname{sbc} O(Z)$ containing $x$, then by definition $(2.1)$, there is $(L, A)$ soft closed set such that $x \in(L, A) \subset(U, A)$. So, by the hypothesis, $(P, A) \cap(L, A) \neq \emptyset$. Hence, $(P, A) \cap(U, A) \neq \emptyset$ for any $s b c-$ open set $(U, A)_{x}$. Therefore, $x \in \operatorname{sbcCl}(P, A)$.

Theorem 3.10. Let $(Z, \tau, A)$ be an $\boldsymbol{S T} \boldsymbol{S}$ and let $(P, A)$ and $(\mathrm{M}, A)$ be soft sets over $Z$. Then

1) $\operatorname{sbcCl}(P, A)$ is the intersection of all $s b c-\operatorname{closed}$ sets which are containing $(P, A)$.

2) $(P, A) \subset \operatorname{sbcCl}(P, A)$.

3) $\operatorname{sbcCl}(P, A)$ is $s b c-$ closed set in $Z$.

4) $(P, A)$ is $s b c-$ closed iff $(P, A)=\operatorname{sbcCl}(P, A)$.

5) $\operatorname{sbcCl}(\operatorname{sbcCl}(P, A))=\operatorname{sbcCl}(P, A)$.

6) $\operatorname{sbcICl}(\widetilde{\varnothing})=\widetilde{\emptyset}$ and $\operatorname{sbcCl}(\widetilde{Z})=\widetilde{Z}$.

7) If $(P, A) \subset(\mathrm{M}, A)$, then $\operatorname{sbcCl}(P, A) \subset \operatorname{sbcCl}(\mathrm{M}, A)$.

8) If $\operatorname{sbcCl}(P, A) \cap \operatorname{sbcCl}(\mathrm{M}, A)=\widetilde{\emptyset}$, then $(P, A) \cap(\mathrm{M}, A)=\widetilde{\emptyset}$.

9) $\operatorname{sbcCl}(P, A) \cup \operatorname{sbcCl}(\mathrm{M}, A) \subset \operatorname{sbcCl}((P, A) \cup(\mathrm{M}, A))$.

10) $\operatorname{sbcCl}((P, A) \cap(\mathrm{M}, A)) \subset \operatorname{sbcCl}(P, A) \cap \operatorname{sbcCl}(\mathrm{M}, A)$.

Proof. It is obvious.

\section{Acknowledgment}

The authors would like to thank Mustansiriyah University (www.uomustansiriyah.edu.iP),

Baghdad, Iraq for its support in the present work.

\section{References}

1. Molodtsov, D. 1999. "Soft set theory-first results," Computers and Mathematics with Applications, 37(4-5): 19-31.

2. Molodtsov, D., Leonov, V.Y. and Kovkov, D.V. 2006. "Soft sets technique and its application",Nechetkie Sistemyi Myagkie Vychisleniya, 1(1): 8-39.

3. Shabir, M. and Naz, M. 2011. "On soft topological spaces". Computers and Mathematics with Applications, 61(7): 1786-1799.

4. Arockiarani and Arokialancy, A. 2013. "Generalized soft g $\beta$-closed sets and soft gs $\beta$-closed sets in soft topological space", International Journal of Mathemat-ical Archive, 4(2): 1-7.

5. Akdag, M. and Ozkan, A. 2014. "Soft $\alpha$-Open Sets and Soft $\alpha$-Continuous Functions". Abstr. Appl. Anal. 2014, Article ID 891341, 7 pages. doi:10.1155/2014/891341. https://projecteuclid.org/euclid.aaa/1412273201.

6. Chen, B. 2013. "Soft Semi-open sets and related properties in soft topological spaces," Applied Mathematics and Information Sciences, 7(1): 287-294.

7. Akdag, M. and Ozkan, A. 2014. "Soft b-open sets and soft b-continuous functions". Math Sci, 8: 124, doi: 10.1007/s40096-014-0124-7.

8. Ibrahim, H. 2013. "Bc-Open Sets in Topological Spaces", Advances in Pure Mathematics, 3(1): 34-40. doi: 10.4236/apm.2013.31007.

9. Zorlutuna, M., Akdag, W. K. and Atmaca, S. 2012."Remarks on soft topological spaces," Annals of Fuzzy Mathematics and Informatics, 3(2): 171-185.

10. Hussain, S. and Ahmad, B. 2015. "Soft separation axioms in soft topological spaces", Hacettepe Journal of Mathematics and Statistics, 44(3): 559 - 568.

11. Selvi, A. and Arockiarani, I. 2015. "On Soft slightly $\pi$ g-continuous functions", Journal of Progressive Research in Mathematics, 3(2): 168-174. 